\title{
Effective in Vitro Synergy of Piperacillin/Tazobactam Plus either Netilmicin or Aztreonam against Metallo- $\beta$-lactamase-producing Pseudomonas Aeruginosa
}

\author{
Wesam Hatem Amer ${ }^{*}$, Marwa Mohamed Ezzat Abd-Elmonsef \\ Medical Microbiology and Immunology Department, Faculty of Medicine, Tanta University, Egypt
}

Copyright $\mathrm{C} 2016$ by authors, all rights reserved. Authors agree that this article remains permanently open access under the terms of the Creative Commons Attribution License 4.0 international License.

\begin{abstract}
This study was designed to compare the activity of piperacillin/tazobactam (TZP) in combination with either netilmicin (NET) or aztreonam (ATM) against metallo- $\beta$ lactamases (M $\beta \mathrm{L}$ ) Pseudomonas aeruginosa. Out of 46 nosocomial Ps. aeruginosa isolates, 38 (82.6\%) isolates were carbapenem-resistant by disc diffusion susceptibility testing. These were then screened by imipenem + EDTA combined disc test for M $\beta \mathrm{L}$ production. Thirty $(78.9 \%)$ M $\beta$ L-producing isolates detected were further tested for antibiotic synergy against NET-TZP and ATM-TZP combinations by Etest synergy and Etest/agar dilution tests. Neither combination showed antagonism. The synergistic effect of NET-TZP combination was detected in [27 (90\%) and 28 (93.3\%), respectively] and ATM-TZP combination in $[24(80 \%)$ and $25(83.3 \%)$, respectively] of the tested isolates by Etest synergy and Etest/agar dilution tests, respectively. Thus, we have determined the synergistic effect of these two combinations for treating $\mathrm{M} \beta \mathrm{L}$-producing Ps. aeruginosa. Significance and Impact of the Study: The present study has shown valuable information on the combination treatment of Ps. aeruginosa and recommends their use for treatment of resistant Ps. aeruginosa.
\end{abstract}

Keywords Pseudomonas Aeruginosa, MBL, Synergy Tests, Netilmicin, Aztreonam, Piperacillin/Tazobactam

\section{Introduction}

Pseudomonas aeruginosa is an important opportunistic pathogen that plays a serious role in many infections especially in immuno-compromised patients [1]. Infections by this organism are characterized as severe and resistant to treatment with drugs [2]. Metallo- $\beta$-lactamase (M $\mathrm{ML})$ production by $P$ s. aeruginosa is a major challenge because it confers multidrug resistance (MDR) to $\beta$-lactam agents, as well as co-resistance to fluoroquinolones and aminoglycosides [3]. Continued emergence and rapid spread of MDR PS. aeruginosa strains have resulted in increased morbidity, mortality and cost $[1,4]$. This increased the need for alternative strategies such as combination of anti-pseudomonal antibiotics as an alternative strategy in the management of these cases.

Although aminoglycosides are among the oldest antibiotics to treat serious infections, they have severe side effects such as nephrotoxicity, ototoxicity (vestibular and auditory) and, rarely, neuromuscular blockade and hypersensitivity reactions. They are concentration-dependent, bactericidal agents that have certain limitations including the age of the patient, serum creatinine level and the duration of exposure $[5,6]$. This and the appearance of new and safer antibiotics resulted in the dramatic drop in the use of aminoglycosides since 1970s. However, their use is being reconsidered again due to the development of MDR among different microorganisms [6]. Netilmicin (NET) is an effective bactericidal drug with a little toxic effect rather than other aminoglycosides which favors its use with severe infections in critical cases that cannot withstand severe toxicity associated with other aminoglycosides [7].

It is well known now that a $\beta$-lactam plus an aminoglycoside is the standard for treating severe Ps. aeruginosa infections [8]. This synergy could be explained by the theory that exposure of bacterial cell to $\beta$-lactam drugs lessen the barriers, leading to increase the susceptibility to aminoglycosides [9]. The effective in vitro synergistic activity of NET plus $\beta$ - lactams against resistant Ps. aeruginosa has been documented by several studies with variable degrees. These combinations included NET plus penicillin [10], piperacillin [11], carbenicillin [12], aztreonam , 3rd generation cephalosporins as cefotaxime, 
cetazidime, cetriaxone and cefoperazone [14].Some of these combinations showed better results than gentamicin when combined with the same $\beta$ - lactams $[11,12,13]$.

On the contrary, aztreonam (ATM) is a monobactam which has a strong activity against Gram-negative rods with no remarkable toxicity $[8,14]$. It may be similar in efficacy to aminoglycosides as gentamicin and tobramycin. It could be an effective alternative to aminoglycosides in serious Gram-negative infections [15].

$\beta$-lactam/ $\beta$-lactamase inhibitor drugs, represented by piperacillin/tazobactam (TZP) is particularly effective against $P$ s. aeruginosa [16].It is effective in the treatment of wide range of infections including moderate to severe polymicrobial infections[17].

In certain conditions, such as severe infection in patients with poor renal functions or immuno-compromised patients who need prolonged courses of treatment, it is preferable to use dual $\beta$-lactam drugs rather than $\beta$-lactamaminoglcoside combination. The mechanism of synergy between $\beta$-lacatams includes the action of different particles at different penicillin binding proteins and subsequent interference of cell wall synthesis. Another mechanism is the synergistic effect of the inhibition by $\beta$-lactamases[18].

The goal of this study was to compare the activity of TZP in combination with either NET or ATM against MßL-producing Ps. aeruginosa using two methods; Etest synergy and Etest/agar dilution.

\section{Material and Methods}

\subsection{Bacterial Isolates and Growth Conditions:}

This study included 46 nosocomial Ps. aeruginosa isolates obtained from Microbiology Laboratory of Infection Control Unit. Pure cultures were preserved on $15 \%$ glycerol in screw top tubes at $-70^{\circ} \mathrm{C}$ until use. Tubes were transported immediately to the Microbiology Laboratory of Microbiology and Immunology Department, Faculty of Medicine for further processing. To recover the bacteria: the frozen surface of the culture was scraped with a sterile inoculating loop, the bacteria that adhere to the loop were streaked onto the surface of plates and the frozen culture was returned to storage at $-70{ }^{\circ} \mathrm{C}$ [19]. Blood agar and MacConkey's agar plates were used for subculture with incubation at $37^{\circ} \mathrm{C}$ for 24 hours. Any growth was identified as $P$ s. aeruginosa by conventional microbiology methods; colony morphology, Gram film and biochemical reactions [20].

Isolates Data were collected retrospectively from patients fulfilling criteria of Centre for Disease Control (CDC) for nosocomial infections [21] and admitted between January 2015 to July 2015 in Tanta University Hospitals, Egypt. These 46 nosocomial Ps. aeruginosa isolates were collected from patients admitted to emergency ICU, pediatric ICU and surgery wards (18, 17and 11 respectively).

\subsection{Antibiotic Susceptibility Testing:}

Antibiotic susceptibility tests were performed using the Kirby-Bauer disc diffusion method and interpreted according to Clinical and Laboratory Standards Institute

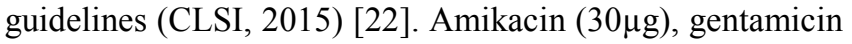
$(10 \mu \mathrm{g})$, NET $(30 \mu \mathrm{g})$, ciprofloxacin $(5 \mu \mathrm{g})$, piperacillin $(100 \mu \mathrm{g})$, TZP $(110 \mu \mathrm{g})$, ceftazidime $(30 \mu \mathrm{g})$, cefepime $(30 \mu \mathrm{gl})$, imipenem $(10 \mu \mathrm{g})$, meropenem $(10 \mu \mathrm{g})$, ATM $(30 \mu \mathrm{g})$, polymyxin-B $(300 \mathrm{U})$ and colistin $(10 \mu \mathrm{g})$ discs (Oxoid, Uk) were used for testing. Ps. aeruginosa ATCC 27853 was used as control strain.

\subsection{Screening Test for MßL:}

Ps. aeruginosa isolates resistant to imipenem or meropenem or both were subjected to the screening test for $\mathrm{M} \beta \mathrm{L}$ production by imipenem (Oxoid, Uk) + EDTA (Zhonglan Industry Co, China) combined disc test as described previously by Yong et al .[23]. Isolates with enhancement of zone size of $\geq 7 \mathrm{~mm}$ between imipenem + EDTA disc compared with imipenem disc alone were considered as M $\beta \mathrm{L}-$ positive. One Ps. aeruginosa strain producing IMP and another one producing VIM that were confirmed by PCR and provided by our laboratory, were used as MBL-positive controls [24]. Ps. aeruginosa ATCC 27853 was used as a M $\beta \mathrm{L}$-negative control.

\subsection{Antibiotic Synergy Testing}

In this study, we chose to use the Etest synergy to measure the in vitro synergistic activity between antibiotic combinations. It is an easy and rapid method with low cost, and it has a high agreement with the time-kill assay test $[25,26]$. In addition, Etest/agar dilution was used to confirm results obtained by the Etest synergy technique $[27,28]$.

\section{Etest Synergy Test:}

Minimum inhibitory concentration (MIC) test strips (Liofilchem ${ }^{\circledR}$, Italy) were used with the following concentrations $(\mu \mathrm{g} / \mathrm{ml})$ : NET, 0.016-256; ATM, 0.016-256; TZP, 0.016-256. Synergy test was measured by Etest synergy method that was determined in triplicate according to Pankey and Ashcraft [25] on Muller-Hinton agar (MHA) (Oxoid, UK) plates. The following antibiotic combinations were tested: NET-TZP and ATM-TZP.

\section{Etest/Agar Dilution Test:}

NET (Merck Schering Plough, Nestpan-Cipla, USA) was incorporated into MHA at concentration of $0.06 \mathrm{mg} / \mathrm{L}$ as previously described $[22,29]$. Plates were inoculated with $0.5 \mathrm{McF}$ arland suspensions of each isolate, then TZP Etest strips were applied. After $24 \mathrm{hrs}$ incubation at $37^{\circ} \mathrm{C}$, the strips were read and the Etest MICs were compared to a series performed in the absence of TZP. The same was done 
with ATM (Bristol Mayers squibb, Princeton, USA) using concentration of $0.5 \mathrm{mg} / \mathrm{L}$ and then TZP Etest strips were applied. In this method, we used the least concentration of the drug to be added to the MHA in case of Ps. aeruginosa $[22,29]$.

The FIC was calculated for each antibiotic in each combination by using the following formula:

FIC of drug $\mathbf{A}=$ (MIC of drug A in combination)/ (MIC of drug A alone)

FIC of drug $\mathbf{B}=($ MIC of drug $\mathrm{B}$ in combination $) /$ (MIC of drug $\mathrm{B}$ alone)

$\boldsymbol{\Sigma}$ FIC $=$ FIC of drug A + FIC of drug B.

Synergy was defined by a $\boldsymbol{\Sigma}$ FIC of $\leq 0.5$. Antagonism was defined by a $\boldsymbol{\Sigma}$ FIC of $>4$. Interactions represented by a $\boldsymbol{\Sigma}$ FIC of $\boldsymbol{\Sigma} 0.5$ but $\leq 4$ were termed indifferent.

MIC required to inhibit $50 \%$ and $90 \%$ of the isolates (MIC50 and MIC90, respectively) were calculated using Microsoft $\Sigma{ }^{\circledR}$ Excel ${ }^{\circledR} 2007$ using Etest strips. Ps. aeruginosa ATCC 27853 was used as control strain.

\subsection{Ethical Consideration}

It was approved by the Ethical Committee of the Faculty of Medicine, Tanta University, Egypt.

\subsection{Statistical Analysis}

Data were analyzed using statistical package of social science (SPSS) version 21. Qualitative data were expressed as frequency and percentage. Chi-square test was used to examine the relation between qualitative variables. $P$-value less than 0.05 was considered significant.

\section{Results}

Out of 46 Ps. aeruginosa isolates tested for their antibiotic susceptibility, eight isolates $(17.4 \%)$ were carbapenem sensitive while thirty eight $(82.6 \%)$ isolates were carbapenem-resistant, among which there were only $30 \mathrm{M} \beta \mathrm{L}$ producers. According to specimen type , 20(43.5\%) of $\mathrm{M} \beta \mathrm{L}$ isolates were from endotracheal aspirates , 7(15.2\%) from wound swabs, and 3(6.5\%) from urine (Table 1).

By disc diffusion method, all $\mathrm{M} \beta \mathrm{L}$ isolates were sensitive for both colistin and polymyxin B, while $(23.3 \%, 10 \%)$ were sensitive for ATM and NET, respectively. Intermediate sensitivity was $(43.3 \%)$ for piperacillin and $(33.3 \%)$ for both TZP and ATM. Absolute resistance (100\%) was found with all other tested antibiotics (Table 2). These results identified our choices for the preferable combinations to be tested.

The MIC ranges for NET, ATM and TZP tested against MßL-producing Ps. aeruginosa strains, and their $\mathrm{MIC}_{50}$ and $\mathrm{MIC}_{90}$ values are shown in (Table 3).

Table 1. Distribution of isolated $P_{S}$. aeruginosa according to department and specimen type.

\begin{tabular}{|c|c|c|c|}
\hline Number(46) & $\begin{array}{c}\text { Carbapenem sensitive Ps. } \\
\text { aeruginosa (8) }\end{array}$ & \multicolumn{2}{|c|}{$\begin{array}{c}\text { Carbapenem resistant Ps. aeruginosa } \\
\text { (38) }\end{array}$} \\
\hline Department & & M $\beta$ L positive & M $\beta$ L negative \\
\hline Emergency ICU & 3 & 12 & 3 \\
\hline Pediatric ICU & 3 & 11 & 3 \\
\hline Surgery wards & 2 & 7 & 2 \\
\hline Specimen & \multicolumn{3}{|c|}{} \\
\hline Endotracheal aspirate & 1 & 20 & 3 \\
\hline Wound & 3 & 7 & 3 \\
\hline Urine & 4 & 3 & \\
\hline
\end{tabular}

ICU: intensive care unit, M $\beta$ L: metallo beta lactamase. These isolates were collected from different departments of Tanta University Hospitals, Egypt

Table 2. Susceptibility pattern of $30 \mathrm{M} \beta \mathrm{L}-$ producing Ps. aeruginosa isolates by disc diffusion method

\begin{tabular}{|c|c|c|c|}
\hline Drug & Sensitive & Intermediate & Resistant \\
\hline Amikacin & $0(0 \%)$ & $0(0 \%)$ & $30(100 \%)$ \\
\hline Netilmicin & $3(10 \%)$ & $0(0 \%)$ & $27(90 \%)$ \\
\hline Gentamicin & $0(0 \%)$ & $0(0 \%)$ & $30(100 \%)$ \\
\hline Ceftazidime & $0(0 \%)$ & $0(0 \%)$ & $30(100 \%)$ \\
\hline Cefepime & $0(0 \%)$ & $0(0 \%)$ & $30(100 \%)$ \\
\hline Ciprofloxacin & $0(0 \%)$ & $0(0 \%)$ & $30(100 \%)$ \\
\hline Colistin & $30(100 \%)$ & $0(0 \%)$ & $0(0 \%)$ \\
\hline Polymyxin B & $30(100 \%)$ & $0(0 \%)$ & $0(0 \%)$ \\
\hline Aztreonam & $7(23.3 \%)$ & $10(33.3 \%)$ & $13(43.3 \%)$ \\
\hline Piperacillin/Tazobactam & $0(0 \%)$ & $10(33.3 \%)$ & $20(66.7 \%)$ \\
\hline Piperacillin & $0(0 \%)$ & $13(43.3 \%)$ & $17(56.7 \%)$ \\
\hline Imipenem & $0(0 \%)$ & $0(0 \%)$ & $30(100 \%)$ \\
\hline Meropenem & $0(0 \%)$ & $0(0 \%)$ & $30(100 \%)$ \\
\hline
\end{tabular}


Table 3. MIC values for NET, ATM and TZP antibiotic tested against $30 \mathrm{M} \beta \mathrm{L}$ - producing Ps. aeruginosa isolates

\begin{tabular}{|c|c|c|c|}
\hline Drug & MIC range $\mu \mathrm{g} / \mathrm{ml}$ & MIC $_{50} \mu \mathrm{g} / \mathrm{ml}$ & MIC $_{90} \mu \mathrm{g} / \mathrm{ml}$ \\
\hline NET & $0.5-256$ & 3 & 32 \\
\hline ATM & $1-32$ & 2 & 8 \\
\hline TZP & $0.5-256$ & 1.5 & 24 \\
\hline
\end{tabular}

NET: netilmicin, ATM: aztreonam, TZP: piperacillin/tazobactam, MIC: minimal inhibitory concentration.

Table 4. Effects of antibiotic combinations tested against $30 \mathrm{M} \beta \mathrm{L}-$ producing Ps. aeruginosa isolates.

\begin{tabular}{|c|c|c|c|c|c|}
\hline Method & $\begin{array}{c}\text { Drug } \\
\text { combination }\end{array}$ & Synergism & Indifference & Antagonism & P-value \\
\hline \multirow{2}{*}{ Etest synergy } & NET- TZP & $27(90 \%)$ & $3(10 \%)$ & $0(0 \%)$ & \multirow{2}{*}{0.424} \\
\hline & ATM- TZP & $24(80 \%)$ & $6(20 \%)$ & $0(0 \%)$ & \\
\hline \multirow{2}{*}{ Etest/agar dilution } & NET- TZP & $28(93.3 \%)$ & $2(6.7 \%)$ & $0(0 \%)$ & \multirow{2}{*}{0.227} \\
\hline & ATM- TZP & $25(83.3 \%)$ & $5(16.7 \%)$ & $0(0 \%)$ & \\
\hline
\end{tabular}

NET : netilmicin, ATM : aztreonam, TZP: piperacillin/tazobactam, $\mathrm{p}<0.05$ significant estimated by chi -square test.

The effects of antibiotic combinations tested against the 30 M $\beta$ L-producing Ps. aeruginosa isolates were assessed using the $\Sigma$ FIC index criteria. There was (90\%, 93.3\%) synergy between NET-TZP and (80\%, 83.3\%) synergy between ATM-TZP by using Etest synergy and Etest/agar dilution test, respectively and there was no antagonism by either two methods (Table 4).

\section{Discussion}

Infection with Ps. aeruginosa is a very serious problem due to difficulties associated with treatment options for resistant isolates and poor response rates of patients. In addition, it is usually recruited from immuno-compromised patients with nosocomial infections. Therefore, special care should be taken while determining its antibiotic susceptibility pattern and prescribing the suitable antibiotic therapy. During the last decades, many resistance enzymes had been detected to be produced by Ps. aeruginosa, including Extended-spectrum $\beta$-lactamases (ESBL), AmpC and $\mathrm{M} \beta \mathrm{L} . \mathrm{M} \beta \mathrm{L}$ enzymes confer resistance to all $\beta$-lactams (except ATM) and usually to aminoglycosides [30]. M $\beta$ L-producing Ps. aeruginosa is an emerging threat as it can hydrolyze carbapenems which are given as a last resort to the patient having infection with ESBL and AmpC-lactamase producing Ps. aeruginosa [31].

In this study, 30 out of $38(78.9 \%)$ carbapenem resistant Ps. aeruginosa isolates were positive for $\mathrm{M} \beta \mathrm{L}$ production by imipenem + EDTA double disk synergy test. This is a relatively high ratio when compared with finding of Abd El-Baky et al [31] where only $31(53.4 \%)$ of their 58 Ps. aeruginosa isolates were $\mathrm{M} \beta \mathrm{L}$ producers, and that of Asghar study [32] who reported that M $\beta$ L-producing Ps. aeruginosa were identified in only $76(15.9 \%)$ of their 478 isolates. While a close result was obtained by Toval et al. [33] where their M $\beta \mathrm{L}$ production ratio was 102 (81.6\%) out of 125 carbapenem resistant Ps. aeruginosa isolates. This high ratios of carbapenem resistance and $\mathrm{M} \beta \mathrm{L}$-producing
Ps. aeruginosa in our institution may be due to absence of antibiotic policy and the misuse of antibiotics especially $\beta$-lactams and carbapenems.

According to antibiotic susceptibility pattern, we noticed that all $\mathrm{M} \beta \mathrm{L}$ positive $P$ s. aeruginosa isolates were sensitive to polymyxin B and colistin. All 30 isolates showed absolute resistant (100\%) to amikacin, gentamicin, ceftazidime, cefepime and ciprofloxacin. While, number of resistant isolates to NET was 27(90\%), TZP 20(66.7\%), piperacillin $17(56.7 \%)$ and ATM $13(43.3 \%)$. These results supported the measurement of the synergistic effect of TZP combined with either NET or ATM on M 3 L-positive isolates.

TZP is a $\beta$-lactam/ $\beta$-lactamase inhibitor combination that has a broad spectrum activity against most Gram-positive and Gram-negative aerobic and anaerobic bacteria [34]. The addition of tazobactam enhances the antimicrobial activity of piperacillin against most $\beta$-lactamase-producing organisms [35]. As regard drug-drug interaction, when aminoglycosides are administered in combination with piperacillin in patients with end-stage renal disease requiring hemodialysis, the concentrations of the aminoglycosides (especially tobramycin) may be significantly altered and should be monitored. Probenecid if administered concomitantly with TZP, it prolongs the half-life of piperacillin by $21 \%$ and that of tazobactam by $71 \%$. In addition, coagulation parameters should be monitored regularly during simultaneous administration of high doses of heparin and oral anticoagulants [36]. TZP is generally safe, well tolerated and of low cost. This renders it to be a reliable option when used in combination with other drugs for the early empiric treatment of moderate-to-severe infections [34,37]. On the other hand, NET is an ethyl derivative of dehydrogenated C1a gentamicin and is active against many gentamicin-resistant organisms. It has the least nephrotoxicity and ototoxicity among the other aminoglycosides [38,39]. Although, some studies did not find NET to be useful against gentamicin-resistant Ps. aeruginosa [40,41]. Others like 
Biswal et al., [42] detected less resistance of Ps. aeruginosa to NET $(58.62 \%)$ when compared with amikacin and gentamicin $(81.03 \%$ for each), which is consistent with our findings.

In the current study, synergy was detected between TZP and NET (90-93.3\%) by Etest synergy and Etest /agar dilution respectively with no antagonism. Several studies measured the synergy between TZP and aminoglycosides like tobramycin, gentamicin and amikacin. As far as we know, this is the first attempt for detecting the in vitro synergy between TZP and NET as one of the aminoglycosides. An early study was performed on the combination between carbenicillin and NET which detected a synergy rate of (45.65\%) in Ps. aeruginosa isolates [40]. Consistent with our study, a clinical trial noted the effectiveness of NET-TZP combination as a safe excellent early empiric therapy for high-risk febrile neutropenia in pediatric cancer patients [43]. Studies on the other aminoglycosides detected synergy between TZP plus either; tobramycin (50\%) [44], amikacin (95.9\%, 42\%) [44,45] respectively , arbekacin (100\%) [3] and gentamicin $(33.33 \%)$ [46].

On the other hand, less synergy was detected between TZP and ATM (80-83.3\%) in this study by Etest synergy and Etest /agar dilution respectively, with no antagonism. Fujimura et al. [3] tested this combination in vitro and reported synergy rate $(89.9 \%)$ with no antagonism, which is similar to our results. Also investigators supported the in vivo and in vitro synergy between ATM and piperacillin alone, even without the tazobactam addition [45,47]. Nevertheless, Pinheiro et al. [48] did not recommend the ATM-TZP combination as the best combination in empirical or definitive treatment against Ps. aeruginosa.

No statistically significant differences in synergy rates were found between the two combinations using both methods $(P$-value $=0.227$ and 0.424$)$. This may refer to the effectiveness of both combinations to overcome $\mathrm{M} \beta \mathrm{L}-$ producing Ps. aeruginosa.

\section{Conclusions}

This study determines that NET-TZP and ATM-TZP combinations were effective against M $\beta \mathrm{L}$-producing PS. aeruginosa. This may confer better choices in empirical therapy, with less toxicity especially in critically ill patients.

\section{Acknowledgements}

We would like to profusely thank the staff of Microbiology laboratory of Infection Control Unit, Faculty of Medicine, Tanta University for their cooperation in the collection of isolates used in this study.

\section{Conflict of Interest}

The authors declare that there is no conflict of interest.

\section{Research Funding}

None.

\section{REFERENCES}

[1] Y.M. Wang, Y.L. Wan, X. Wang, P.Y.Wang, Q.S.Wang, X.L. $\mathrm{Cao}$ and Y.C.Liu. The mutL mutation in Pseudomonas aeruginosa isolates reveals multidrug-resistant traits and possible evolutionary trends. J Int Med Res 38(6), 2011-2022, 2010 .

[2] J.A.Driscoll, S.L. Brody and M.H. Kollef. The epidemiology, pathogenesis and treatment of Pseudomonas aeruginosa infections. Drugs 67, 351-368, 2007.

[3] S. Fujimura, H. Takane, Y.Nakano and A. Watanabe. In vitro synergy studies based on tazobactam/piperacillin against clinical isolates of metallo-beta-lactamase-producing Pseudomonas aeruginosa. J Antimicrob Chemother 64(5), 1115-1116, 2009.

[4] N.C. Gordon, K. Png and D.W Wareham. Potent synergy and sustained bactericidal activity of a vancomycin-colistin combination versus multidrug-resistant strains of Acinetobacter baumannii. Antimicrob Agents Chemother 54(12), 5316-5322, 2010.

[5] L.S. Gonzalez and J.P. Spencer. Aminoglycosides: a practical review. Am Fam Physician 58(8), 1811-1820, 1998.

[6] K.A. Wargo and J.D. Edwards. Aminoglycoside-Induced Nephrotoxicity. J pharm pract 27(6), 573-577, 2014.

[7] D.M. Campoli-Richards, S. Chaplin, R.H. Sayce and K.L. Goa. Netilmicin. A review of its antibacterial activity, pharmacokinetic properties and therapeutic use. Drugs. Nov; 38(5):703-56, 1989.

[8] D.S. Burgess and S. Nathisuwan. Cefepime, piperacillin/tazobactam, gentamicin, ciprofloxacin, and levofloxacin alone and in combination against Pseudomonas aeruginosa. Diagn Microbiol Infect Dis 44(1), 35-41, 2002.

[9] L. G. Burman, K.Nordström, and G.D. Bloom . Murein and the Outer Penetration Barrier of Escherichia coli K-12, Proteus mirabilis, and Pseudomonas aeruginosa. J Bacteriol.; 112(3): 1364-1374, 1972.

[10] E. Jawetz .Combined antibiotic action: some definitions and correlations between laboratory and clinical results. Antimicrob. Agents Chemother, 203-209, 1967.

[11] K. Machka, W. Baierl and I. Braveny. Comparative synergistic activity of netilmicin-piperacillin versus gentamicin-piperacillin. Eur J Clin Microbiol. Aug; 1(4): 238-42, 1982.

[12] K. P. FU and H. C. NEU. InVitro Synergistic Effect of Netilmicin, a New Aminoglycoside Antibiotic. Antimicrob Agents Chemother. Sep; 10(3): 511-518, 1976.

[13] H. Giamarellou, N. P. Zissis, G. Tagari and J. Bouzos. In Vitro Synergistic Activities of Aminoglycosides and New, 
B-Lactams Against Multiresistant Pseudomonas aeruginosa. Antimicrob Agents Chemother., Apr.; 25(4) : 534-536, 1984.

[14] H.C. Neu. Aztreonam activity, pharmacology, and clinical uses. Am. J. Med 88 (Suppl. 3c), 2-6, 1990.

[15] R.N. Brogden and R.C. Heel. Aztreonam. A review of its antibacterial activity, pharmacokinetic properties and therapeutic use. Drugs 31(2) 96-130, 1986.

[16] K. A. Toussaint and J. C. Gallagher, $\beta$-Lactam $/ \beta$-Lactamase Inhibitor Combinations: From Then to Now .Ann Pharmacother January 49 (1): 86-98, 2015.

[17] H.M. Bryson and R.N. Brogden .Piperacillin/tazobactam. A review of its antibacterial activity, pharmacokinetic properties and therapeutic potential. Drugs. Mar; 47(3):506-35, 1994.

[18] S. J .Pedler and A. J .Bint . Combinations of beta lactam antibiotics. Br Med J (Clin Res Ed). Apr 7; 288(6423): 1022 1024, 1984.

[19] L. F. Gibson and J. T. Khoury. Storage and survival of bacteria by ultra-freeze. Lett. Appl. Microbiol., June 3 (6) :127-129, 1986.

[20] J.R. Govan. Pseudomonas aeruginosa. In: Collee G, Barrie PM, Andrew PF, Anthony S, editors. Mackie and McCartne Practical Medical Microbiology. 14th edn. New York: Churchill Livingstone; 413-424, 2006.

[21] J.S. Garner, W.R. Jarvis, T.G. Emori, T.C. Horan and J.M. Hughes. CDC definitions for nosocomial infections. Am J Infect Control 16, 128-140, 1988.

[22] Clinical and Laboratory Standards Institute (CLSI 2015). Performance Standards for Antimicrobial Susceptibility Testing. 25th informational supplement, CLSI document M100-S25. Wayne, PA: CLASI; 2015.

[23] D.Yong, K. Lee, J.H. Yum, H.B. Shin, G.M. Rossolini and Y.Chong. Imipenem-EDTA disk method for differentiation of metallo-beta-lactamase producing clinical isolates of Pseudomonas spp. and Acinetobacter spp. J Clin Microbiol 40, 3798-3801, 2002.

[24] Y. S.Yu, T. T. Qu, J. Y. Zhou, J. Wang, H. Y. Li, and T. R. Walsh. Integrons containing the VIM-2 metallo- $\beta$-lactamase gene among imipenem-resistant Pseudomonas aeruginosa strains from different Chinese hospitals. J Clin Microbiol 444242-245, 2006.

[25] G.A. Pankey and D.S. Ashcraft. In Vitro Synergy of Ciprofloxacin and Gatifloxacin against Ciprofloxacin-Resistant Pseudomonas aeruginosa. Antimicrob Agents Chemother 49(7), 2959- 2964, 2005.

[26] G.A. Pankey, D.S. Ashcraft, and A. Dornelles. Comparison of 3 Etest methods and time-kill assay for determination of antimicrobial synergy against carbapenemase-producing Klebsiella species. Diagn Microbiol Infect Dis 77, 220-226, 2013.

[27] D.W. Wareham and D.C. Bean. In-vitro activity of polymyxin $\mathrm{B}$ in combination with imipenem, rifampicin and azithromycin versus multidrug resistant strains of Acinetobacter baumannii producing OXA-23 carbapenemases. Ann Clin Microbiol Antimicrob, 5: 10, 2006.
[28] Hornsey, C. Longshaw, L. Phee and D.W. Wareham. In Vitro Activity of Telavancin in Combination with Colistin versus Gram-Negative Bacterial Pathogens. Antimicrob Agents Chemother; 56(6): 3080-3085, 2012.

[29] JM Andrews. Determination of minimum inhibitory concentration. Antimicrob Chemother 48 (suppl 1): 5-16, 2001 .

[30] P. Sacha, P.Wieczorek, T. Hauschild, M. Zórawski, D. Olszańska and E.Tryniszewska. Metallo-beta-lactamases of Pseudomonas aeruginosa--a novel mechanism resistance to beta-lactam antibiotics. Folia Histochem Cytobiol 46(2), 137-142, 2008

[31] R.M. Abd El-Baky, N.H. Abd El-Azeim and G.F.M .Gad. Prevalence of Extended-Spectrum Beta-Lactamase, AmpC Beta- Lactamase, and Metallo-Beta-Lactamase among Clinical Isolates of Pseudomonas aeruginosa. J Adv Biotechnol Bioeng 1(1), 22-29, 2013.

[32] A.H. Asghar. Antimicrobial susceptibility and metallo- $\beta$-lactamase production among Pseudomonas aeruginosa isolated from Makkah Hospitals. Pak J Med Sci.; 28(5), 781-786, 2012.

[33] F.Toval, A. Guzmán-Marte, V. Madriz, T. Somogyi, C. Rodríguez, F. García. Predominance of carbapenem-resistant Pseudomonas aeruginosa isolates carrying blaIMP and blaVIM metallo- $\beta$-lactamases in a major hospital in Costa Rica. J Med Microbiol 64 (Pt 1), 37-43, 2015.

[34] M.Young and G.L. Plosker. Piperacillin/tazobactam: a pharmacoeconomic review of its use in moderate to severe bacterial infections. Pharmacoeconomics 19(11), 1135-1175, 2001.

[35] L.L. Schoonover, D.J. Occhipinti, K.A. Rodvold and L.H. Danziger. Piperacillin/tazobactam: a new beta-lactam/beta-la ctamase inhibitor combination. Ann Pharmacother 29(5), 501-514, 1995

[36] FDA Wyeth Pharmaceuticals Inc. W10414C006 Philadelphia, PA 19101 ET01 Rev 07/05www.fda.gov/ohrms/dockets/doc kets/.

[37] A.Gin, L. Dilay, J.A. Karlowsky, A. Walkty, E.Rubinstein and G.G. Zhanel. Piperacillin-tazobactam: abeta-lactam/beta -lactamase inhibitor combination. Expert Rev Anti Infect Ther 5(3), 365-383, 2007.

[38] A.M. Lerner, M.P. Reyes, L.A. Cone, D.C. Blair, W. W. Jansen, G.E. Wright and R.R. Lorber. Randomised, controlled trial of the comparative efficacy, auditory toxicity, and nephrotoxicity of tobramycin and netilmicin. Lancet 1(8334), 1123-1126, 1983.

[39] E.J. Begg and M.L. Barclay. Aminoglycosides: 50 years on. Br J Clin Pharmacol 39, 597-603, 1995.

[40] D.J. Briedis and H.G. Robson. Comparative activity of netilmicin, gentamicin, amikacin, and tobramycin against Pseudomonas aeruginosa and Enterobacteriaceae. Antimicrob Agents Chemother 10(4), 592-597, 1976.

[41] J.J. Rahal, M.S. Simberkoff, K. Kagan, and N.H. Moldover. Bactericidal efficacy of Sch 20569 and amikacin against gentamicin-sensitive and -resistant organisms. Antimicrob Agents Chemother 9(4), 595-599, 1976.

[42] I. Biswal, B.S. Arora, D. Kasana, Neetushree. Incidence of 
multidrug resistant Pseudomonas aeruginosa isolated from burn patients and environment of teaching institution. J Clin Diagn Res 8(5), DC26-29, 2014.

[43] S. Aksoylar, N. Cetingül, M. Kantar, D. Karapinar, K. Kavakli and S. Kansoy. Meropenem plus amikacin versus piperacillin-tazobactam plus netilmicin as empiric therapy for high-risk febrile neutropenia in children. Pediatr Hematol Oncol 21(2), 115-123, 2004.

[44] D. Dundar and M. Otkun. In-vitro efficacy of synergistic antibiotic combinations in multidrug resistant Pseudomonas aeruginosa strains. Yonsei Med J 51(1), 111-6, 2010.

[45] D.S. Burgess and R.W. Hastings. Activity of piperacillin/tazobactam in combination with amikacin, ciprofloxacin, and trovafloxacin against Pseudomonas aeruginosa by time-kill. Diagn Microbiol Infect Dis 38(1), $37-41,2000$.

[46] I.M. Gould and K. Milne. In-vitro pharmacodynamic studies of piperacillin/tazobactam with gentamicin and ciprofloxacin. J Antimicrob Chemother 39(1), 53-61, 1997.

[47] Y.H. Chen, P.L. Lu, J.J. Tsai, T.P. Chen and C.F. Penga. In Vitro Activities of Antibiotic Combinations against Clinical Isolates of Pseudomonas Aeruginosa. Kaohsiung J Med Sci 20(6), 261-267, 2004

[48] M.R., Pinheiro, H.R. Lacerda, R.G. Melo, and M.A. Maciel. Pseudomonas aeruginosa infections: factors relating to mortality with emphasis on resistance pattern and antimicrobial treatment. Braz J Infect Dis 12(6), 509-515, 2008. 